\title{
Analysis of evolution of NOAA 7912 active region on 19 October 1995
}

\author{
P. Rudawy ${ }^{1}$, L. van Driel-Gesztelyi ${ }^{2,3}$, B. Cader-Sroka ${ }^{1}$, P. Mein ${ }^{2}$, N. Mein ${ }^{2}$, B. Schmieder ${ }^{2}$, J.-M. Malherbe $^{2}$, and \\ B. Rompolt ${ }^{1}$ \\ 1 Astronomical Institute of the Wroclaw University, PL 51622 Wroclaw, ul Kopernika 11, Poland \\ 2 Observatoire de Paris, Section de Meudon, F-92195 Meudon Principal Cedex, France \\ ${ }^{3}$ Konkoly Observatory, Budapest, Pf. 67, H-1525, Hungary
}

Received January 28; accepted June 18, 1999

\begin{abstract}
An analysis of a minor (B3.2) flare related to an X-ray jet in the reversed polarity NOAA 7912 active region is presented, focusing on various kinds of activity observed in the $\mathrm{H} \alpha$ line with the MSDP instrument. Using complementary Yohkoh soft X-ray observations and a Kitt Peak magnetic field map we study the influence of a rather low and graduate energy release on filaments, fibrils and chromospheric brightenings and their relevant coronal features. We find that this small flare affected the entire AR and was associated with strong up- and downflows along remote fibrils and filaments, which appear to be connected by large-scale loops. It is shown that at least one of the observed brightenings was caused by downflow of the matter, thus, by the kinematic heating of the material. The filament in the vicinity of the flare footpoints changed the most: it broke into two parts, temporarily rose to higher altitudes inside the AR and even erupted outside of the AR, re-forming only three hours later. We show that even a minor flare can lead to important morphological and dynamical changes in an active region.
\end{abstract}

Key words: Sun: chromosphere — Sun: activity — Sun: filaments

\section{Introduction}

On 19 October 1995, during the coordinated international observing campaign, we obtained a long time series of observations of the NOAA 7912 active region with the French Multichannel Subtractive Double Pass spectrograph (MSDP) mounted at the German Vacuum Tower Telescope (VTT) on Tenerife. We also used images taken with Yohkoh Soft X-ray Telescope (SXT). In the investigated active region two X-ray jets were observed with the

Send offprint requests to: P. Rudawy
Yohkoh/SXT at 10:29 UT and 16:59 UT which had major response at radio wavelengths (type III bursts) (van Driel-Gesztelyi et al. 1998a; Schmieder et al. 1998). The jets were related to two small flares (GOES levels B3.2 and $\mathrm{B} 1.3$ and $1 \mathrm{~B}$ and $1 \mathrm{~N}$ importance in $\mathrm{H} \alpha$, respectively).

Jets are normally related to relatively small chromospheric flare events, while their impact in the corona and the interplanetary space may be much more important than that of confined flares of much higher chromospheric importance. Normally, jets occur in regions with high shear and certain magnetic complexity: at one of their footpoints there is frequently found an included, or parasitic polarity.

Spectral observations being at our disposal allow us to investigate the kinematics of material motions in the specified active phenomena. We analyze what kind of magnetic and chromospheric features correspond to footpoints of $\mathrm{X}$-ray bright loops and how the chromospheric structures evolve due to the flare and jet activity.

Most of the formerly published papers on complex analysis of active regions' evolution concerned the regions displaying high flare activity. The main aim of this paper is to analyze the activity evolution in a region of medium activity in order to investigate the influence of rather low energy release on filaments, fibrils and chromospheric brightenings.

\section{Observations}

\subsection{Ho data}

Observations in $\mathrm{H} \alpha$ line performed by P. Mein, J.-M. Malherbe and J. Staiger with the 9-channel MSDP (Mein et al. 1996) started at 08:15 UT and ended at 13:17 UT. The MSDP scanned a rectangular area on the solar disc of $2^{\prime} \times 2.4^{\prime}$ (duration of one scan was about three minutes), except for the period between 10:18 UT and 10:50 UT when the observed area was reduced to about $2^{\prime} \times 0.6^{\prime}$ in order to attain higher time resolution (here one scan lasted 


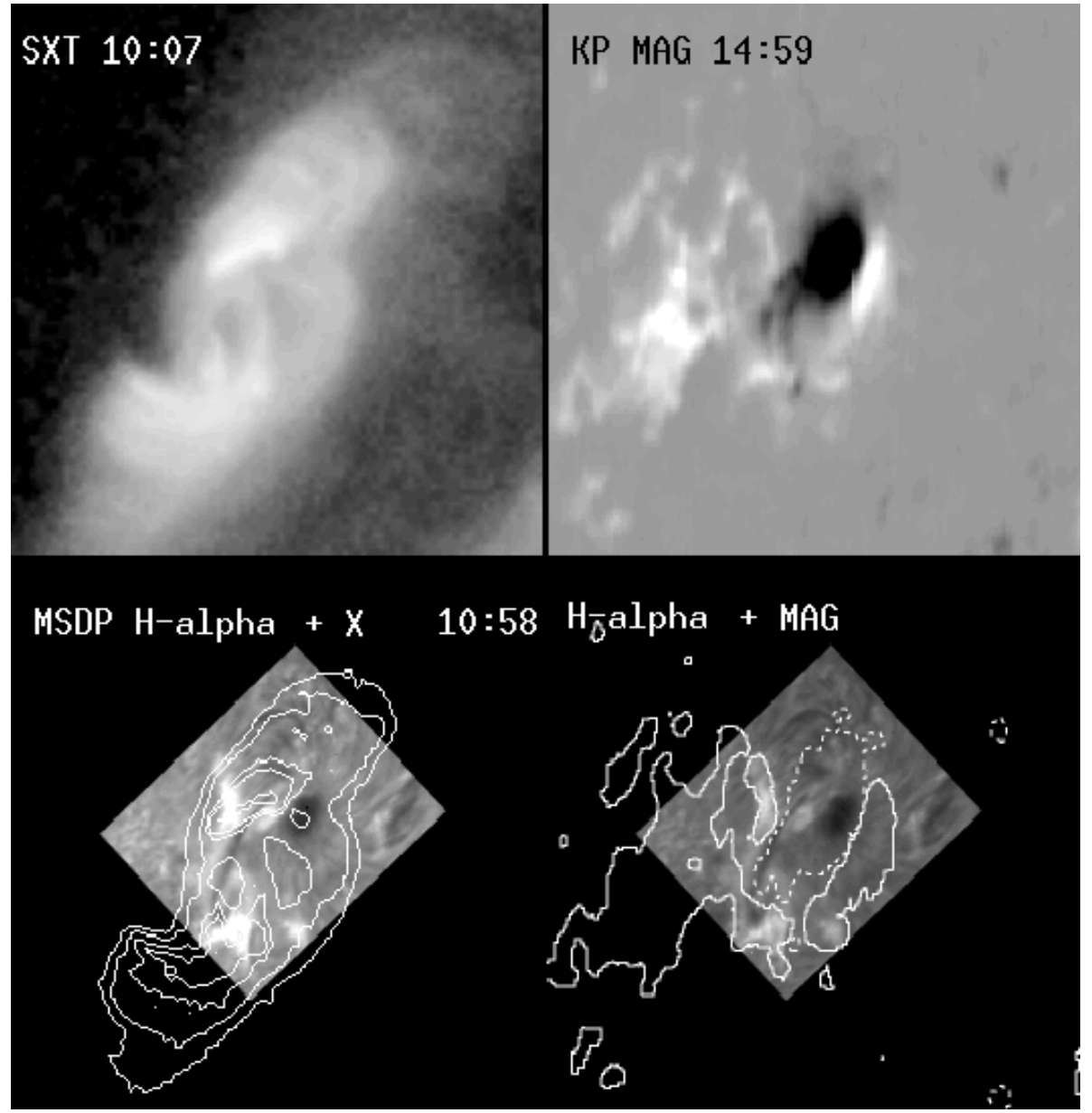

Fig. 1. Yohkoh/SXT observation and magnetic fields of NOAA 7912 active region co-aligned with $\mathrm{MSDP} \mathrm{H} \alpha$ image on 19 October 1995. Upper left panel: X-ray image taken at 10:07 UT; upper right panel: Kitt Peak magnetogram taken at 14:59 UT; lower left panel: $\mathrm{H} \alpha$ image at 10:58 UT with the X-ray contour; lower right panel: $\mathrm{H} \alpha$ image at 10:58 UT with the 50 Gauss magnetic field contours
45 seconds only). During the time of observations the active region was located at about $\mathrm{S} 10 \mathrm{~W} 50$. The seeing was not stable and oscillated between good and medium.

The active region area chosen for the final analysis was of about $1.6^{\prime} \times 2.1^{\prime}$ except for the period $10: 18 \mathrm{UT}-10$ : 50 UT when it was limited to about $1.9^{\prime} \times 0.6^{\prime}$. Processing of the observational data with the use of special codes written by P. Mein (Mein 1991; Roudier et al. 1991) was made at Meudon Observatory. The $\mathrm{H} \alpha$ line profile was reconstructed at each spatial point as well as intensity maps and Doppler velocities were obtained at various wavelengths along the line profile (up to $\pm 1 \AA$ from the line centre). We used intensity and velocity maps calculated by the bisector method and images at various wavelengths. Further results concerning down- and up-flows of the matter were derived by taking into account the geometrical effects of the location of the active region close to the solar limb.

\subsection{X-ray observations and co-alignment method}

Soft X-ray observations of AR 7912 have been taken with the SXT instrument on board of the Yohkoh spacecraft (Ogawara et al. 1991; Tsuneta et al. 1991) in the framework of the coordinated observing campaign with the MSDP. The high-resolution mode (2.46 arcsec/pixel) field of view of the instrument covered the AR 7912 during the whole MSDP observing period (see Fig. 1, upper left panel). The SXT images have been run through the standard Yohkoh data reduction procedures (SXT-PREP) and then short- and long-exposure images were combined in order to remove the saturation effect and display $\mathrm{X}$-ray structures in a broader brightness range. For the physical analysis of the events we co-aligned the X-ray observations with the twelve times higher resolution (0.2 arcsec/pixel) MSDP images (see Fig. 1, lower left panel). The co-alignment was carried out in several steps using full-disc SXT images and a full-disc Kitt-Peak magnetogram rotated to the time of the X-ray images (see Fig. 1, right panels). Then, the MSDP images, which have no pointing information, were co-aligned with the magnetic map using feature tracking technique. One of the problems of the coalignment was the time-difference between the magnetic map and the $\mathrm{H} \alpha$ observations, which implies a possibility of some differences in the topology due to evolution of the observed structures.

\subsection{Magnetic data}

In an attempt to understand the magnetic topology of the region as well as the flare and jet event, we utilize a full-disc, full resolution magnetogram from Kitt Peak 


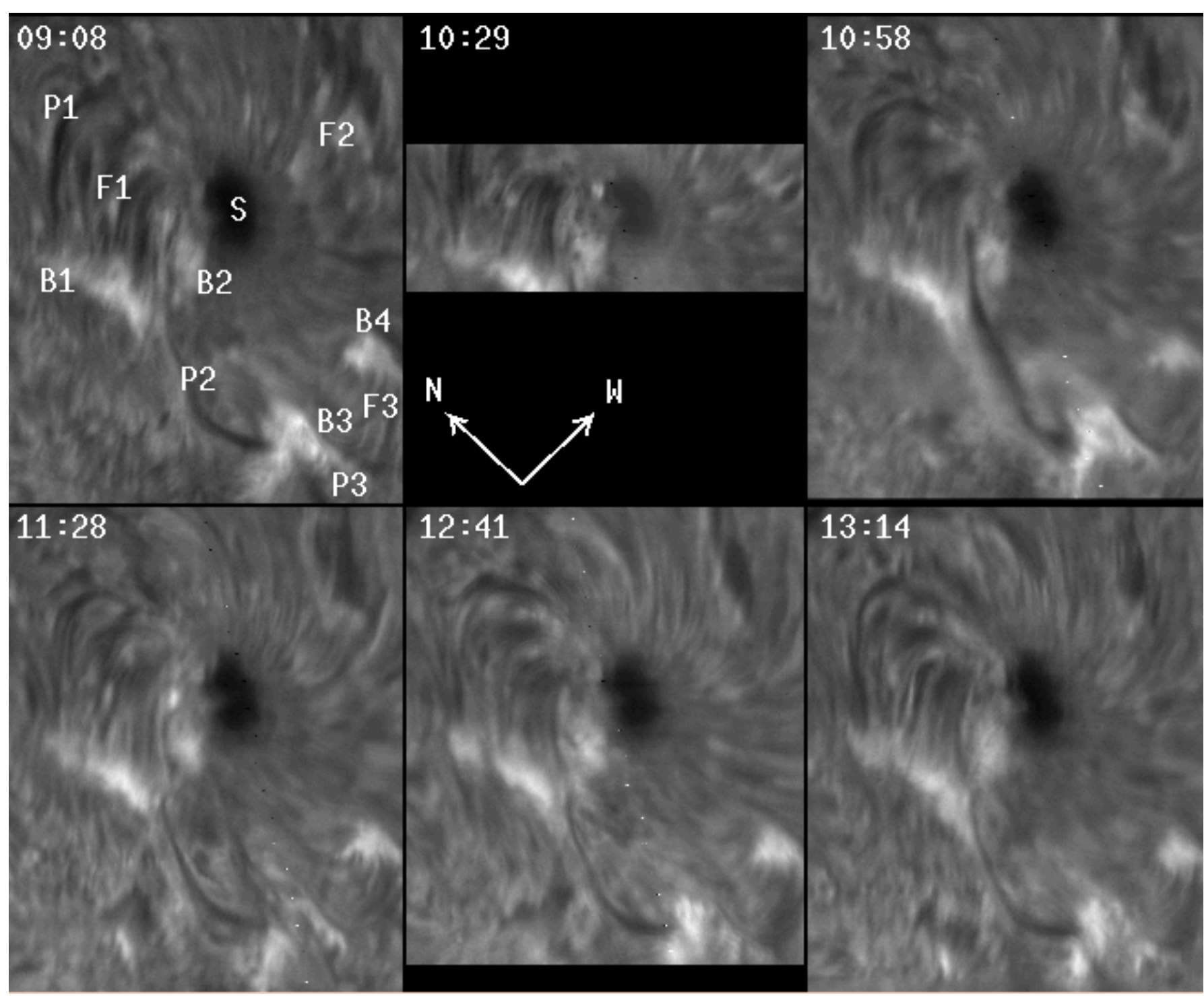

Fig. 2. H $\alpha$ line centre intensity maps of NOAA 7912 active region observed on 19 October 1995 from 09:08 UT to 13:14 UT. The labelled structures are described in the text. The maps cover an area of $2^{\prime} \times 2{ }^{\prime} \cdot 4$ on the solar disc, except for the map obtained at 10:29 UT, when the observed area was reduced to $2^{\prime} \times 0.6$

Observatory, courtesy of K.L. Harvey. The magnetogram was taken on 19 October at 14:59 UT with a resolution of $1.15 \mathrm{arcsec} /$ pixel (see Fig. 1, upper right panel).

\section{Description of the observed features}

\subsection{Ho structures}

From the beginning of the observational period several features of different types were visible in the analyzed active region: (i) a big spot (S) located a little above the centre of the field of view (see Fig. 2); (ii) filaments: a curved filament or a strong fibril (P1) situated to the north-east of the spot, a long filament (P2) running from the eastern periphery of the spot to the south up to a brightening (B3, described below) and a very southern filament (P3) running from B3 to the south; (iii) brightenings: the most important brightenings in the region were located to the east of the spot. The first brightening (B1) was located in an elongated region at the eastern base of anchorage of fibrils F1. The second one (B2) lays to the west of brightening B1, just between the northern part of filament P2 and the spot $\mathrm{S}$. Brightening B2 was situated in the area of the eastern group of the superpenumbral fine filaments. There were also two significant brightenings B3 and B4 situated to the south of the spot S; (iv) three systems of fibrils, the first one (F1) was situated more or less to the north-east of the spot, the second one (F2) consisting of a group of spot's superpenumbral fibrils was located to the west of the spot. The third system of fibrils (F3) was situated to the south of the spot. The eastern legs of fibrils F3 seem to be anchored in the area close to brightening B3 while the western ones at the other brightening B4.

In some high resolution pictures the brightening B3 displayed well visible fibril structure oriented perpendicularly to the P2 and P3 filaments axes. The brightening 


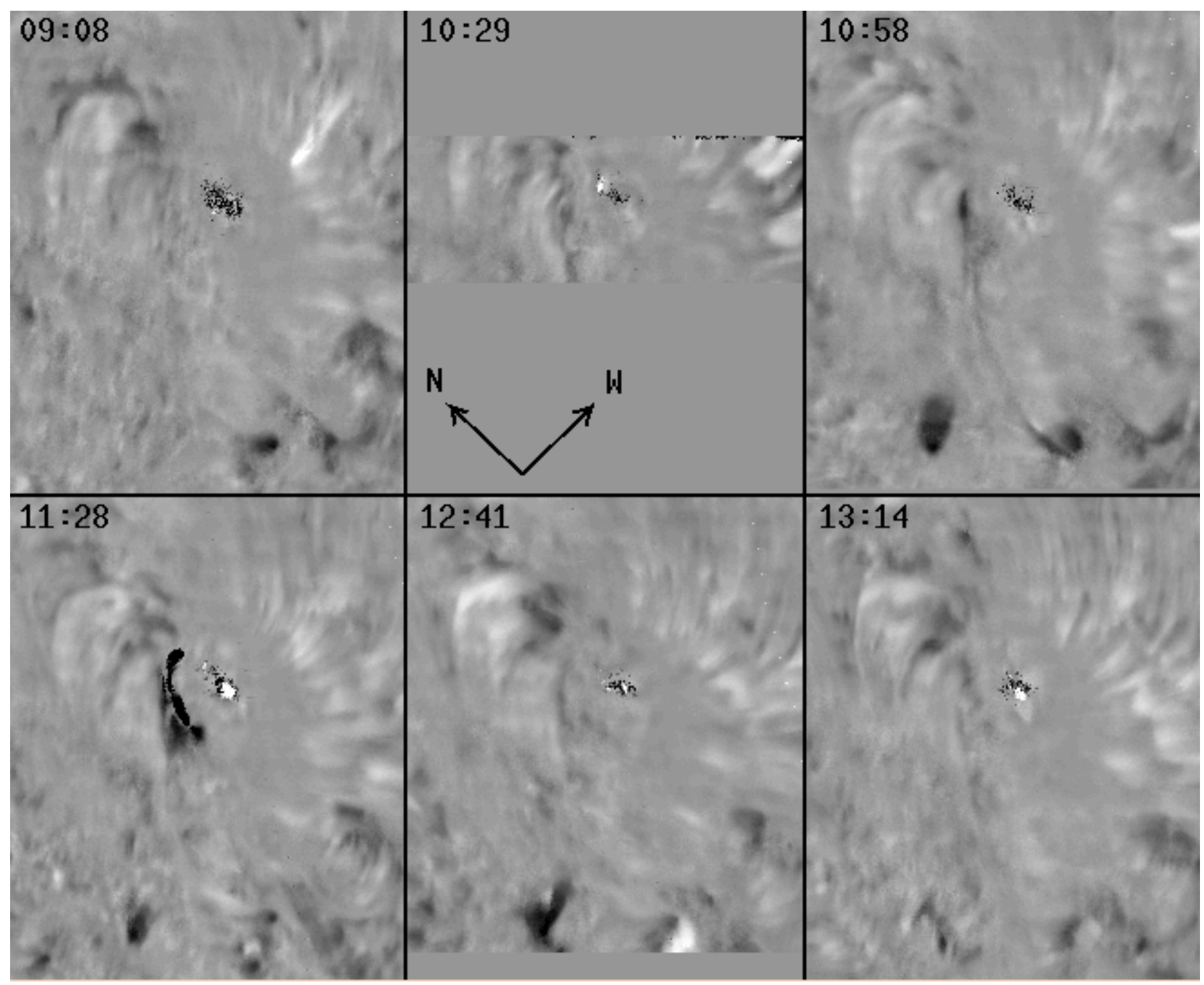

Fig. 3. H $\alpha$ line of sight velocity maps of the AR on 19 October 1995 from 09:08 UT to 13:14 UT. The darkest features correspond to redshifts of $20 \mathrm{~km} \mathrm{~s}^{-1}$ and higher while the brightest ones correspond to blueshifts of $20 \mathrm{~km} \mathrm{~s}^{-1}$ and higher. The Doppler velocities cannot be computed at some points (e.g. in the umbral region), because of the flat shapes of the line profiles

B3, located between filaments P2 and P3, seems to be an important region connecting these two filaments.

At 10:29 UT a B3.2 class flare started in the NOAA 7912 active region, to the south-east of the spot S. MSDP images taken in the red and blue wings of the $\mathrm{H} \alpha$ line at $\pm 0.78 \AA$ reveal a highly dynamical response of the chromosphere to the flare. The line of sight velocity maps Fig. 3) show several regions with velocities toward the observer (blueshifts) or from the observer (redshifts), well related to certain structures of the active region. Detailed description of the evolution of the $\mathrm{H} \alpha$ structures is given in Sect. 4.1.

\subsection{Magnetic fields}

The active region NOAA 7912 consisted of a round, leading spot of negative polarity and a dispersed following area of positive polarity (Fig. 4a). A few small parasitic polarity spots disturbed the bipolar structure of the region (van Driel-Gesztelyi et al. 1997; Schmieder et al. 1998; van Driel-Gesztelyi et al. 1998). During the 22nd solar activity cycle, leading spots of the south hemisphere had positive polarity. Thus, NOAA 7912 located at S12, did not follow Hale's law, but had inversed polarity. The magnetic field was highly sheared as indicated by the clockwise vortex fibril pattern (see Fig. 2, fibrils systems F1 and F2) around the leading spot at the chromospheric level, as well as by the shape and connectivity of coronal loops, which out of two sets of loops formed a "forward S" shape - both pointing to the presence of positive helicity (Pevtsov et al. 1997).

\subsection{X-ray structures}

The soft X-ray loops had a highly sheared appearance, which is not surprising in case of an inversed polarity 

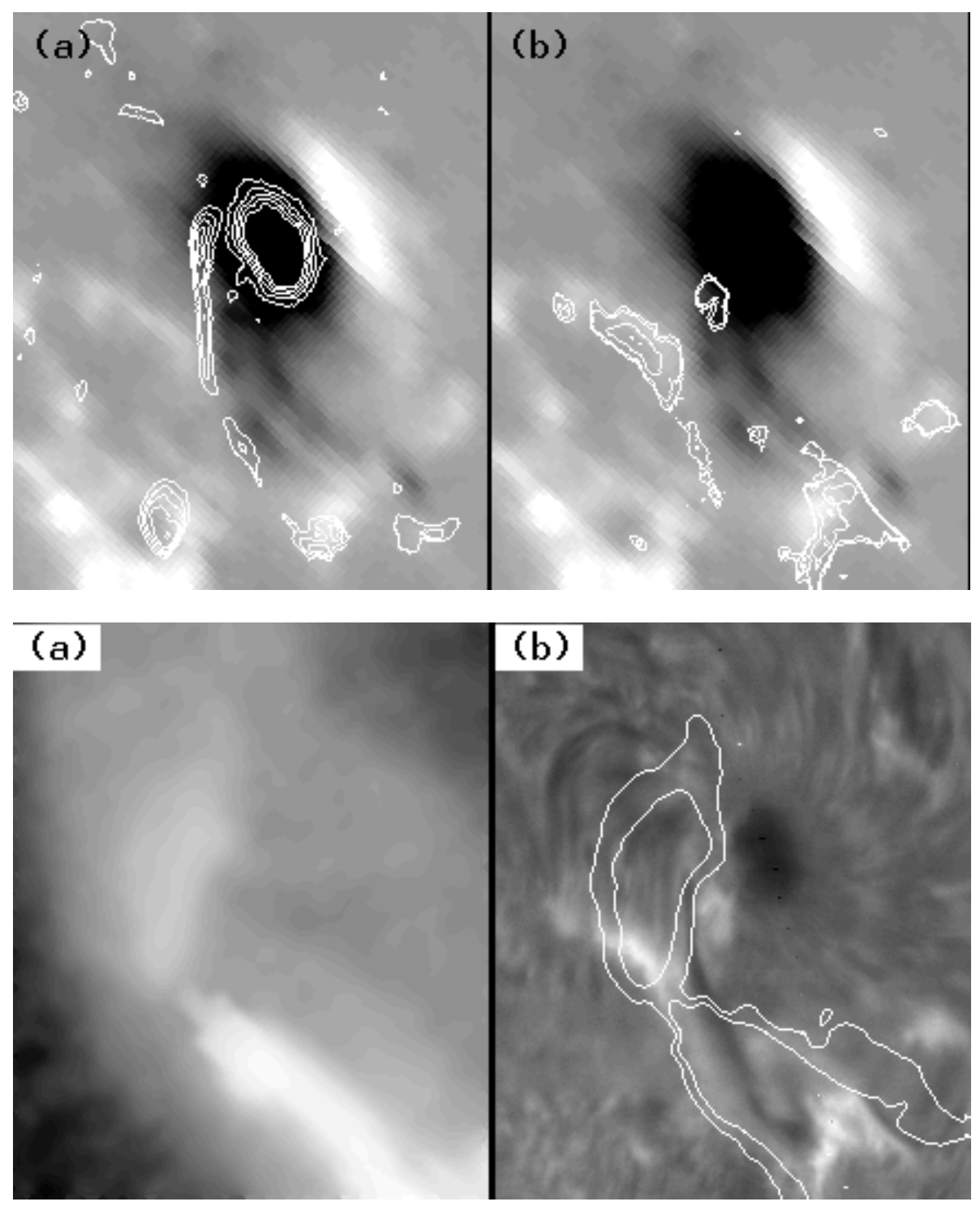

Fig. 4. NOAA $7912 \quad \mathrm{H} \alpha$ structures at 10:58 UT and magnetic fields at 14:59 UT rotated in accordance with the 10:58 UT H $\alpha$ image orientation. a) Magnetic fields and absorbing features seen in the $\mathrm{H} \alpha$ line wing $(+0.78 \AA)$; b) magnetic fields and bright features seen in the $\mathrm{H} \alpha$ line center

Fig. 5. X-ray loops and $\mathrm{H} \alpha$ structures: a) Yohkoh/SXT full resolution image taken at 10:27 UT; b) $\mathrm{H} \alpha$ line centre structures at 10:58 UT and SXR contours at 10:27 UT active region. One set of loops connected the south and south-western parts of the penumbra of the leader spot $\mathrm{S}$ with the south-east part of the region, where the strongest positive polarity was located (cf. Figs. 1 and 4). Another set of loops connected the north-western penumbra with the weak positive polarity concentrations in the north-east part of the AR (Figs. 4 and 5). The brightest stationary $\mathrm{X}$-ray loop visible to the north of the leading spot $\mathrm{S}$ was rooted in the penumbra of the negative magnetic polarity spot and in the $\mathrm{H} \alpha$ brightening region of positive magnetic polarity (B1 region in Fig. 2). This bright loop was above the fibrils F1 (Fig. 5b).

At 10:29 UT the GOES satellite recorded an onset of a B3.2 class flare, which occurred to the south-east of the spot S. The flare was accompanied by a spectacular jet event, which reached a length of $2.5-310^{5} \mathrm{~km}$ toward the south (van Driel-Gesztelyi et al. 1998a). The X-ray jet had its footpoints in the vicinity of the filament P2, where small areas of various polarities created a mixed-polarity region (cf. Figs. 4 and 5).

\section{Evolution of the different structures due to the flare and jet}

\subsection{Detailed description of the chromospheric evolution}

At 10:29:17 UT (during the flare maximum) a significant increase of B1 brightness as well as fainter increase of the B2 brightness was observed. In the spot's penumbra, very close to the northern part of the umbra, a small, point-like brightening, formed by two short comma-like structures was seen (see Fig. 2). In various fibrils of F2 system high redshifts and blueshifts were observed. Around the time of the flare maximum the region of high blueshifts (V1) situated to the west of the spot, started to enlarge its area (see Fig. 3, image at 10:29 UT). V1, being very likely a region of strong upflow of the matter, reached the biggest area and the highest velocities about 10:37 UT. Then V1 
started to decrease and it disappeared about 10:45 UT (see Fig. 3, image at 10:58 UT). Above the northern end of filament P2 there were several small, elongated regions of redshifts. They were also signatures of the material flowing down to the chromosphere along the fibrils forming this end part of filament P2. At the north end of filament P2 small regions of fast downflows were present and were still observed at the southern leg of P2.

At 10:58:30 UT, after the flare which ended at 10:43 UT, the intensity of B1 was still very high, a local increase of the B2 brightness was remarkable and the emission of B3 was strongly increased in comparison with the emission at 09:08:54 UT. The velocities detected in fibrils F2 were much smaller than previously, a significant blueshifts were only present close to the southern part of that region. There were strong redshifts at both ends of filament P2 being evidence for efficient downflow of the matter. A new, quite big region of very strong redshifts (V2) appeared to the east of the southern end of filament P2 (see Fig. 3, image at 10:58 UT). That region was not related to any noteworthy structure visible in the $\mathrm{H} \alpha$ line centre except for a small brightening spatially correlated with the area of the highest (larger than $20 \mathrm{~km} \mathrm{~s}^{-1}$ ) redshifts (see Fig. 3).

Later on, at 11:28:30 UT the east part of the filament $\mathrm{P} 1$ and northern part of P2 were hardly visible. A strong downflow (V3) was present in the northern part of P2. At the southern end of filament P2, near B3, high redshifts were still present. A small, ephemeral bright region appeared to the north of brightening B2. It was situated close to the west border of the strong redshifts region in the northern part of filament P2. The brightness of B4 was significantly enhanced. This region corresponded to the well visible western parts of the fibrils F3, displaying some redshifts. The small brightening in V2 region was more prominent than previously. The area of $\mathrm{V} 2$ region was smaller than at 10:58:30 UT while redshifts in the whole region were still very high. Some superpenumbral fibrils of F2 showed blueshifts related to the often observed inflow of material into the spot. On the basis of comparison of the $\mathrm{H} \alpha$ line centre images and the corresponding velocity maps, taking into account the geometrical effect of active region location with respect to the line-of-sight, we came to a conclusion that fibrils forming F1 were inclined to the solar surface with their tops tilted towards the solar equator. From the careful investigation of the line of sight velocity maps, which show the velocities between $-5 \mathrm{~km} \mathrm{~s}^{-1}$ and $+5 \mathrm{~km} \mathrm{~s}^{-1}$, it is well seen, that the material flowed along the fibrils forming F1 from their north-west to their south-east legs. Moreover, the brightening B1 is spatially correlated with this downflow region.

At 12:41:26 UT the brightness of all the B-regions described previously was more or less the same as at 11:28:30 UT except for the disappearance of the ephemeral brightening seen at 11:28:30 UT and significant enhancement of B3 brilliance. The northern part of filament P2 was well visible again, while a region of redshift present at the same location was reduced to the narrow strip along the filament with much smaller velocities. Redshifts near the brightening B3, at the south end of filament P2 were still high, and, what is interesting, in the same region both, from and toward the observer velocities were present. Some fibrils of F2 still showed blueshifts in the parts located at the place of anchorage of the superpenumbral fibrils close to the penumbra outer border. Blueshifts were also visible in some F1 fibrils. The area of $\mathrm{V} 2$ region increased while its brightness became weaker.

At 13:14:08 UT almost all redshifts became smaller except of B3 brightening, where the velocities stayed the same. Once again, there were higher redshifts in the western part of fibrils F2 at the vicinity of the outer superpenumbral border of the spot. Then, owing to the larger area recorded, filament P3 was well visible again.

The filaments P2 and P3, previously clearly anchored in B3, form now a continuous rope seen quite well in projection above the brightening B3. The leg of the filament $\mathrm{P} 2+\mathrm{P} 3$ anchored in the area of B3 expanded to a long, arch-shaped filament running now above the brightening B3. V3 was almost not visible at that moment. It reached its maximum area, after long gradual increase, at about 11:25 UT. After that time V3 very quickly disappeared. In the velocity map from 12:41 UT stronger redshifts in the region of west legs of $\mathrm{P} 1$ anchorage were visible again. A new region of strong redshifts appeared in the vicinity of former V2. All the bright B-areas became smaller although their brightness stayed the same. It looks as if the overall activity of the NOAA 7912 active region was gradually going down.

\subsection{Summary of the observed changes in $H \alpha$ and $X$-rays}

The flare and jet in X-rays appeared above the P2 filament. Footpoints of the jet and related flare loops are close to B1 and B3 brightenings (Fig. 2, image at 09:08 UT). The brightness of both B1 and B3 increased after the flare.

The filament/fibril changed considerably after the flare. Strong velocities appeared all along it. There was a shift in its position between 09:08 UT and 10:29 UT, consistent with a temporary rise of its middle part. One hour after the flare, the filament appears broken in two in the vicinity of B1. Around that position, at 10:58 UT the line-of-sight velocities showed a double structure already (Fig. 3). In the red and blue wing images these two parts appear differently: upflow is stronger in the southern part and downflow is dominant along the northern part. The downflow was already present at the time of the flare maximum at 10:29 UT. Filament P3, which is the south continuation of $\mathrm{P} 2$, disappeared at the time of the flare and jet (compare images at 09:08 UT and 10:58 UT in Fig. 2), and needed almost three hours to re-form (see Fig. 2, image at 13:14 UT). Possibly it erupted during the event, providing material for the jet. Downflows in 
filament pointing to the sunspots have been previously observed by e.g. Mouradian et al. (1988).

Strong blueshift appeared along the fibril F2 at the time of the flare at the western part of the big spot. SXR loops rooted in $\mathrm{F} 2$ region appear to connect to the region around B1 brightening and also to the northern part of the positive polarity, where we observe a strong downflow (V2 in Fig. 3, image at 10:58 UT).

\section{Discussion and conclusions}

Using spectral scans taken co-temporarily and co-spatially with the 9-channel MSDP instrument in the $\mathrm{H} \alpha$ line, we document four hours of the evolution of the NOAA 7912 active region on 19 October 1995. Utilizing Yohkoh/SXT images co-aligned carefully with the $\mathrm{H} \alpha$ observations we relate the chromospheric features to the coronal ones.

In the quiet state of this reversed polarity $\mathrm{AR}$ the brightest, highly sheared X-ray loops (they were almost parallel to the filament) appeared above a system of strong dark chromospheric fibrils connecting the penumbra of the big leading spot and a bright chromospheric area. These fibrils did not show the characteristic flow pattern of arch filament systems, so we conclude that they did not belong to emerging flux. It is interesting to note that all the brightenings, except for the B2, occurred at the base of anchorage of the feet of the corresponding fibrils systems. The brightest X-ray loop was rooted in the brightest part of the chromospheric plage, in the vicinity of a local (positive) magnetic field maximum. Apart from the time of flare and jet event, this X-ray loop remained the brightest $\mathrm{X}$-ray feature in the AR for several hours.

The studied B3.2 flare associated with the X-ray jet affected the entire AR. Strong up and downflows appeared at both sides of the big leading spot, far away from the flare. The remote fibrils appear to be connected by long sheared coronal loops. The important downflow of the material (V2) was associated with a minor brightening in the $\mathrm{H} \alpha$ line centre, thus the impact of the matter very probably created some chromospheric heating. The flare footpoints were observed at both sides of a filament. This filament changed the most considerably, as expected, due to the flare. Inside the AR the filament separated into two parts, the one close to the flare footpoints appeared temporarily at a higher altitude after the flare. Similar behaviour of a filament after an X-flare was observed by Dezső et al. (1980). It is remarkable that even a minor B3.2 flare can have such effect. There were important velocities observed along the filament during and even half an hour after the flare. The two parts appeared dynamically different: one, located close to the big spot, was dominated by downflow, another - located close to the flare footpoints, by upflow. The south continuation of the filament disappeared, probably erupted, at the time of the flare, and re-formed only about three hours later. The fact, that the filament did not erupt inside the AR can be attributed to the stabilizing effect of the strong overlying loops, while outside of the AR such loops did not prevent the eruption. Such duality in the reaction of a filament to a flare was reported earlier by Raadu et al. (1988) and van DrielGesztelyi et al. (1998b). Raadu et al. (1988) related the activity of the filament to photospheric motion of pores corresponding to change in the small scale magnetic polarity pattern. Such behaviour could be explained with the new MHD models of prominences which show the direct relationship between prominence foot and the presence of small parasitic polarities in the filament channel (Aulanier \& Démoulin 1998; Aulanier et al. 1998 and 1999). Any disturbance in the magnetic field pattern (cancellation, emerging flux) close to a foot could lead to eruption of the foot and more generally of the filament itself.

The presented series of high-resolution spectroscopic $\mathrm{H} \alpha$ observations demonstrated how dynamical is the chromosphere and even a minor flare can lead to important morphological and kinematic changes.

Acknowledgements. This work was supported by the FrenchPolish grant of "Le Conseiller pour la Science et la Technologie" and KBN, No. 6039 (1996). MSDP observations were obtained in the framework of the International time offered by the CCI of the Canarian Observatories and supported by the European Commission through the Access to the Large-Scale Facility "Activity of the Human Capital and Mobility Programme". Thanks are due to J. Staiger, C. Coutard, R. Hellier, the technical team of the VTT Telescope and the Teide Observatory, for their efficient assistance during the campaign. We thank the Yohkoh Team and the YDAC at Mullard Space Science Lab. for the SXT data. The NSO/Kitt Peak magnetogram data used in the paper were kindly provided us by Dr. Karen L. Harvey, the data are produced co-operatively by NSF/NOAO, NASA, GSFC, and NOAA/SEL. LvDG acknowledges the research grant OTKA T026165 and AKP 97-58 2,2. BR and PR were supported by KBN grants no. P03D.025.09 and 2P03D.005.15.

\section{References}

Aulanier G., Démoulin P., 1998, A\&A 329, 1125

Aulanier G., Démoulin P., van Driel-Gesztelyi L., Mein P., DeForest C., 1998, A\&A 335, 309

Aulanier G., Démoulin P., Mein N., van Driel-Gesztelyi L., Mein P., Schmieder B., 1998, A\&A (in press)

Dezső L., Gesztelyi L., Kondás L., Kovács Á., Rostás S., 1980, Solar Phys. 67, 317

Mein N., Mein P., Heinzel P., Vial J.C., Malherbe J.M., Staiger J., 1996, A\&A 309, 275

Mein P., 1991, A\&A 248, 669

Mouradian Z., Martres M.J., Soru-Escaut I., 1988, A\&A 199, 318

Ogawara Y., Takano T., Kato T., Kosugi T., Tsuneta S., Watanabe T., Kondo I., Uchida Y., 1991, Solar Phys. 136, 1

Pevtsov A.A., Canfield R.C., McClymont A.N., 1997, Astrophys. J. 481, 973

Raadu M.A., Schmieder B., Mein N., Gesztelyi L., 1988, A\&A 197,289 
Roudier T., Vigneau J., Mein P., Malherbe J.M., Coutard C., Hellier R., Muller R., 1991, A\&A 248, 237

Schmieder B., van Driel-Gesztelyi L., Mein N., Mein P., Willson R., Raoult A., 1998, Proceedings of an International Meeting held in Guadeloupe, DOM, France, 23-26 February 1998, ESA SP-421, May 1998, p. 157

Tsuneta S., Acton L., Bruner M., et al., 1991, Solar Phys. 136, 37

van Driel-Gesztelyi L., Baranyi T., Mein N., et al., 1997, in Antalová A. and Kučera A. (eds.) JOSO Annual Report '97, Astronomical Institute, Tatranská Lomnica, Slovakia, p. 103

van Driel-Gesztelyi L., Willson R.F., Kile J.N., et al., 1998a, in Donahue B. and Bookbinder J. (eds.) Proc. 10th Cambridge Workshop on Cool Stars, Stellar Systems and the Sun, ASP Conf. Ser. 154, 707

van Driel-Gesztelyi L., Nitta N., Leka K.D, Wülser J.P., 1998b (submitted to Solar Phys.)

van Driel-Gesztelyi L., Schmieder B., Baranyi T., 1998c, "Evolution of the Source Region of the Interplanetary Cloud of 18-20 Oct. 1995", Adv. Space Res. (submitted) 